\title{
Immunolabelling of Isolated Cell Fractions
}

\author{
H. Cook*, A. Warley*, K. Brady* E Vermeulen ** A. Jen** and R. Morris** \\ *CUI King's College London, New Hunt's House, Guy's Campus London SE1 1UL, UK \\ ** Wolfson CARD, King's College London
}

Biochemical reactions are frequently studied in isolated cell fractions, but there is a need to confirm the composition of the fraction and this is frequently achieved by electron microscopy. Immunolocalization of the antigen of interest within that fraction would offer further proof of the association of a particular antigen or enzyme within the given cell fraction. We have adapted immunochemical methods to confirm the presence of glycoproteins in ribosomal fractions isolated from rat brain.

Pellets of isolated ribosomes were fixed for conventional electron microscopy in 100mM PIPES-Cl $\mathrm{pH} 7.4,2 \mathrm{mM} \mathrm{MgCl}_{2}, 1 \%$ paraformaldehyde and $0.1 \%$ glutaraldehyde, for buffer. The pellets were dehydrated through an ascending series of alcohol and embedded in Spurr's resin. The pellets were sectioned and examined on a Hitachi $\mathrm{H} 7600$ electron microscope. For immunolabelling, pellets of ribosomes were lightly fixed in 4\% formaldehyde in PIPES buffer. After 30 minutes the pellet was resuspended in warm $\left(37^{\circ} \mathrm{C}\right)$ gelatine $(10 \%$ in PIPES buffer $)$ and concentrated by centrifugation in a microfuge. The microfuge tube was placed on ice for 30 mins and the tip of the tube containing the pellet cut off with a razor blade. The solidified pellet was removed and cut into small cubes. These were infiltrated with $2.3 \mathrm{M}$ sucrose overnight before being cryofixed by plunging into liquid nitrogen. Cryosections, $70 \mathrm{~nm}$ thick, were cut from the pellets using a RMC CRX cryoultramicrotome. The sections were retrieved on droplets of $2.3 \mathrm{M}$ sucrose and transferred to 100 mesh Pioloform-coated Ni EM grids. Immunolabelling was carried out after the technique of Tokuyasu using wheatgerm agglutinin labelled with $10 \mathrm{~nm}$ colloidal gold to detect $\mathrm{N}$-acteyl glucosamine containing glycoproteins [1].

Conventional electron microscopy showed that the cell fractions contained both rough and smooth vesicles (Fig 1 A). Material that has been prepared for immunolabelling (Fig 1B) showed that the labelled lectin is associated with smooth vesicles as would be expected for proteins in the later stages of glycosylation. This work shows that immunolabelling methods can be adapted successfully for the study of cell fractions.

\section{References}

[1] K.T.Tokuyasu J. Microsc. 143 (1986) 139.

[2] This work was funded by BBSRC BB/C506680/1 

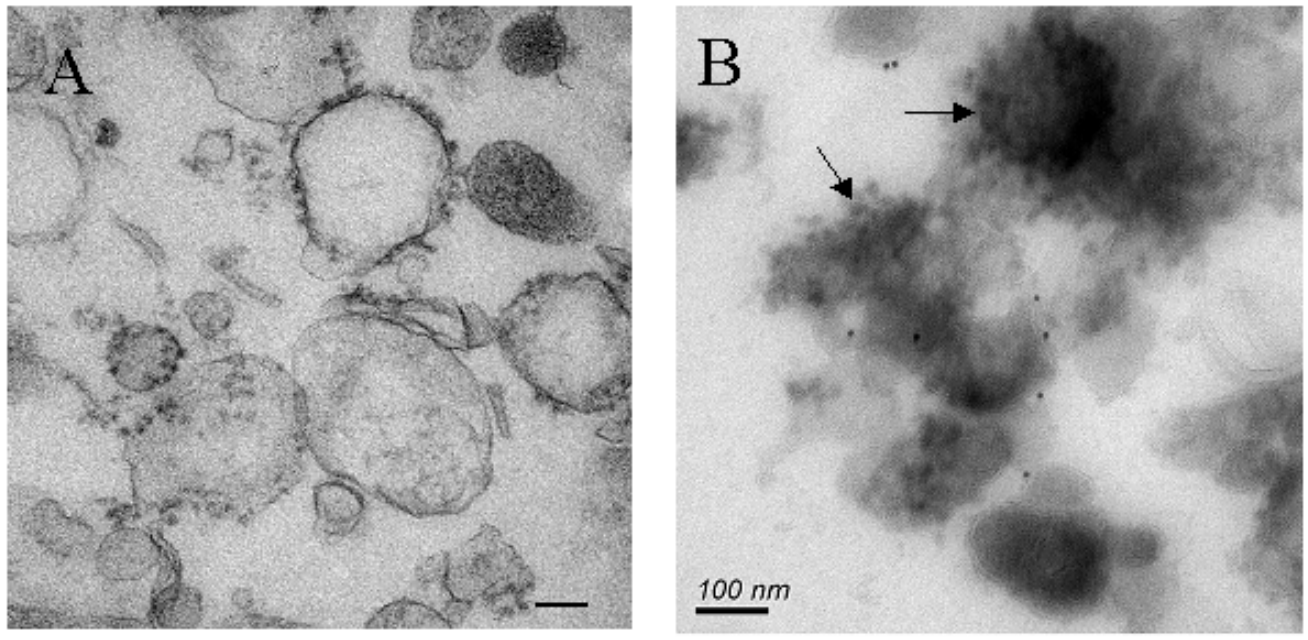

Figure $1 \mathrm{~A}$ ) EM of isolated microsomal fraction from rat brain showing that the pellet is composed of both rough and smooth vesicles. B) Isolated microsomes that have been prepared for immunoEM and labelled with wheat germ agglutinin. Although both rough (arrows ) and smooth vesicles are present the labelling is confined to vesicles with a smooth membrane profile. Marker $=100 \mathrm{~nm}$ 